\title{
Psychiatrische Regeldienste und multikulturelle Realität
}

Meryam Schouler-Ocak

\author{
Akademisches Lehrkrankenhaus der Georg-August-Universität Göttingen
}

psychoneuro 2003; 29 (12): 582-585

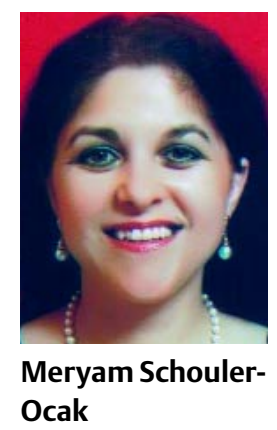

Obwohl seit mehreren Jahrzehnten Menschen mit Migrationshintergrund zum Alltag im psychiatrischen Versorgungssystem gehören, ist ihre Beratung, Betreuung und Behandlung nach wie vor defizitär. Durch die interkulturelle Öffnung der Versorgungseinrichtungen könnte ein wichtiger Schritt in Richtung Integration vollzogen werden. Eine unverzichtbare Voraussetzung stellt dabei die interkulturelle Kompetenz dar. Ziel sollte sein, allen Patienten mit den gleichen einheitlichen Standards zu begegnen.

$\mathrm{N}$ eben Arbeitsmigranten erster, zweiter, dritter und vierter Generation sind aus Osteuropa stammende Aussiedlerpopulationen, Bürgerkriegsflüchtlinge, Asylantragsteller und traumatisierte Flüchtlinge sowie Menschen ohne Aufenthaltsstatus zu nennen. Ende 2001 lebten insgesamt 7,3 Mio. Migranten in Deutschland. Dies entspricht einem Anteil von knapp 9\% an der Gesamtbevölkerung. Von diesen stammt jeder vierte Migrant aus einem Mitgliedsland der Europäischen Union (1). Der Begriff Migrant meint in diesem Kontext Menschen, die nach Deutschland zugewandert sind, oder direkte Nachkommen dieser Zuwanderer, die in der Regel den gleichen kulturellen Hintergrund tradiert bekommen. Zu nennen sind hier u.a. türkeistämmige Zuwanderer in dritter Generation. Das heißt auch, dass nicht alle Migranten im Besitz einer ausländischen Nationalität sind wie z.B. eingebürgerte Arbeitsmigranten oder deutschstämmige Übersiedler. Kurzgefasst sind Migranten Menschen mit Migrationshintergrund.

\section{Migrationsprozess}

Fast alle o. g. Menschen durchlaufen den Migrationsprozess, den Sluzki (14) in einem Modell, das einen relativ hohen Grad an kulturübergreifender Validität besitzt, darstellt. Dabei unterteilt Sluzki den Migrationsprozess in:

- die Vorbereitungsphase

- den Migrationsakt

- die Phase der Überkompensierung

- die Phase der Dekompensation

- die Phase der generationsübergreifenden Anpassungsprozesse.

Nach diesem Modell wenden sich Menschen mit Migrationshintergrund insbesondere in der Phase der Dekompensation, oft zu spät, gelegentlich gar nicht an die Einrichtungen des Gesundheits- bzw. Versorgungssystems.

\section{Welchen Stressfaktoren sind Migranten ausgesetzt?}

Zu den Belastungsfaktoren bzw. psychosozialen Stressoren, die eben zu dieser Dekompensation führen können, zählen u.a.:
- aufenthaltsrechtliche und arbeitsrechtliche Belastungen

- wenig planbare Zukunftsperspektiven

- Erleben von Ausgegrenztsein

- Bevormundung, Ablehnung und fehlende Wertschätzung durch die Mehrheitsbevölkerung

- Trennungserfahrungen und Verlustgefühle, Heimweh

- Auflösung von Familienverbänden, Vereinsamung, Isolation, Rollenverlust und -diffusion

- schlechtere Qualifikation in Schule und Beruf

- Arbeitslosigkeit, geringe Anteilnahme am Arbeitsleben, Armut

- ungünstige Wohn- und Arbeitsbedingungen

- schlechtere gesundheitliche Versorgung

- geringe Anteilnahme an gesellschaftlichen Ereignissen der Mehrheitsbevölkerung

- Versagens- und Insuffizienzgefühle

- Kommunikationsschwierigkeiten.

Diese Faktoren können krankmachend wirken und/oder umgekehrt die Genesung verzögern. Sie sind jedoch nicht per se als Krankheitsursache einzustufen.

Brucks berichtet, dass gerade im Bereich der Prävention, Gesundheitsaufklärung und Gesundheitsbildung eine Unterversorgung be- 
steht. Im Bereich nicht indizierter Medikamente dagegen besteht eine Überversorgung (2). Insgesamt werden durch zu hohe Zugangsbarrieren chronische Patientenkarrieren gefördert. Folge können eine erlernte Hilflosigkeit sein (2).

Als Gründe für die mangelnde Inanspruchnahme dieser Systeme können genannt werden u.a.:

- Informationsmangel, unzureichende Kenntnisse über die Strukturen des Gesundheitssystems

- fehlende bzw. unzureichende interkulturelle Verständigungsmöglichkeiten

- Stigmatisierung, Scham, Diskriminierungserfahrungen

- Finanzierungsunsicherheit

- Qualifikations- und Kompetenzmängel bei den Mitarbeitern der psychiatrisch - psychosozialen Versorgungssysteme

- Defizite in der Gesundheitsaufklärung.

Die von uns in der Institutsambulanz des Niedersächsischen Landeskrankenhauses in Hildesheim seit 1996 häufig beobachteten psychiatrischen Krankheitsbilder bei Migranten sind aus dem Spektrum der

- Affektiven Störungen (F30-F39)

- Neurotischen, Belastungs- und somatoformen Störungen (F40-F48)

- Psychischen und Verhaltensstörungen durch psychotrope Substanzen (F10-F19)

- Persönlichkeits- und Verhaltensstörungen (F60-F69) $(12,13)$.

\section{Kulturelle Besonderheiten und psychische Erkrankungen:}

Unterschiedliche kulturelle Besonderheiten können bereits beim Erstkontakt, bei dem Begrüßungsritual, auffallen (9). So z.B. in:

- Italien: Gestikulieren, laut sprechen, Stille ist peinlich; beim Treffen Händeschütteln, Wangenkuss, Körperkontakt.

- China: Man küsst sich nicht, auch ein Ehepaar küsst sich nicht vor anderen Leuten.

- Vietnam: Kopfnicken, kein Körperkontakt (9).
Bei den eigentlichen psychiatrischen Krankheitsbildern sind die Symptome kulturübergreifend, die Bedeutung der einzelnen Symptome und der Umgang mit ihnen ist jedoch kulturgebunden (4). Ein Beispiel stellt die religiöse Wahnvorstellung dar. Je nach religiöser $\mathrm{Zu}-$ gehörigkeit kann sich der Patient als den dazu gehörigen Propheten Jesus, Mohammed, Buddha o.Ä. wähnen.

\section{Kasuistik 1}

Herr A. stammt aus der Türkei, aus Anatolien, ist der zweite Sohn von vier Geschwistern. Die Familie lebte von der Landwirtschaft. Als er eingeschult wurde, ging zunächst der Vater, kurze Zeit später auch die Mutter als Arbeitsmigranten nach Deutschland. Einige Jahre später folgte er mit seinem Bruder den Eltern nach. In Deutschland schaffte er mit Mühe und Not den Hauptschulabschluss. Er fand keine Lehrstelle, besuchte eine Berufsvorbereitungsklasse und begann dort mit dem Konsum von verschiedenen Substanzen, hauptsächlich jedoch Cannabis. Er fiel auf durch Rückzug, Isolation, ständiges Beten, Rezitieren aus dem Koran, beschimpfte die Familie, dass sie nicht gläubig sei. Eines Tages fing er in seinem Zimmer an herumzuschreien, zerschlug Gegenstände, redete, schimpfte laut vor sich. Alle Hilfsangebote lehnte er ab. Auf der Grundlage des Nds. PsychKG wurde er eingewiesen und behandelt. Es bestand bei ihm eine paranoid-halluzinatorische Psychose, möglicherweise drogeninduziert.

Herr A. gab an, sich als Gesandten Allahs zu fühlen und durch seine Gebete ihm zu huldigen. Später berichtete er, auch die Stimmen des Teufels gehört zu haben, mit dem er gekämpft habe.

Ein anderes Beispiel verdeutlicht den kulturgebundenen Umgang mit Ehre und Scham. In diesem Konzept sind namus und seref, Ehre und Scham, enthalten. Namus ist ein Begriff für die sexuelle Integrität vor allem der Frauen. Die Familienehre wird durch die Keuchheit und Enthaltsamkeit der weiblichen Familienmitglieder aufrechterhalten. Die Familienehre, namus, hat einen wichtigen Platz im Leben dieser Menschen. In diesem Zusammensein hat jeder die Pflicht, in Würde sich zu verhalten und die Ehre und damit das Ansehen der Familie nicht zu verletzen (11). Bei Verlust der Ehre z.B. durch sexuelle voreheliche Beziehung der Tochter können Tragödien entstehen.

\section{Kasuistik 2}

Frau B. ist kurdischer Abstammung, spricht gut türkisch. Sie stammt aus Südostanatolien in der Türkei. Als jüngste Tochter von sechs Geschwistern mit drei Brüdern und zwei Schwestern wuchs sie in sehr ärmlichen Verhältnissen auf. Das Dorf, in dem ihre Familie lebte, habe sich genau in der Front zu den kurdischen Separatisten befunden. Sie habe sehr viel Gewalt mitangesehen. Als der Vater getötet worden sei, seien die Geschwister von dort auf unterschiedlichsten Wegen geflohen. Frau B. sei später von ihren Brüdern nach Deutschland nachgeholt worden. Auf dem Wege nach Deutschland sei sie von verschiedenen Männern vergewaltigt worden. In Deutschland habe sie einen Asylantrag gestellt. Hier angekommen, sei sie nicht in der Lage gewesen, sich jemanden anzuvertrauen. Als Frau B. 18 Jahre alt geworden sei, planten die Brüder, sie mit einem kurdischen Landsmann zu verheiraten. Die Vorbereitungen begannen. Frau B. entwickelte Alpträume, Angst- und Unruhezustände, Erregungsausbrüche, Ohnmachtsanfälle, Hyperventilationsanfälle. Je näher der Hochzeitstermin kam, desto schlechter sei es Frau B. ergangen, bis sie schließlich einen Suizidversuch mit Medikamenten unternahm. In einem Allgemeinkrankenhaus wurde die Akutbehandlung durchgeführt und im Anschluss daran wurde sie in der Psychiatrie bei uns vorgestellt.

Bereits im Erstgespräch schilderte sie unter Tränen, was ihr widerfahren war und was sie zu erwarten hatte. Sie war durch die Vergewaltigungen keine Jungfrau mehr und hatte panische Ängste vor der Hochzeitsnacht und der Schande, die sie damit ihrer Familie bringen würde. Sie hatte Angst um ihre Zu- 
kunft und auch um ihr Leben. In ihrer Heimat spiele namus und seref eine sehr große Rolle. Viele würden lieber sterben als in Schande weiterleben.

Menschen aus dem mediterranen Raum erleben sich meist als Ganzes von der Krankheit ergriffen. Sie machen häufig Angaben wie „alles kaputt“ (3, 4, 7,10). Sie können auch Organchiffren benutzen wie z.B. der Magen falle, die Leber zerfalle, womit sinngemäß mitgeteilt wird „Ich halte es nicht mehr aus, die Belastung wird mir zuviel“ (3, 10). Diese Organchiffren können als Krankheitskonstrukte angesehen werden, indem einerseits Belastung signalisiert wird, andererseits je nach körperlichem Symptom eine psychologische Erklärung innerhalb der kulturellen Gruppe impliziert wird, die außerhalb dieses Kreises in ihrer emotionalen Dimension nicht verstanden wird.

Eine ähnliche Symbolik lässt sich auch im z.B. türkisch-griechischen Kulturkreis finden. Ein ausgeprägtes Engegefühl im Brustkorb (gr. stenochoria) beschreibt sowohl seelische Belastung als auch Trauer. Das Wort nervös (gr. nerva) stellt eine somatisierte Ausdrucksform von Angst und Depression dar (10). Nicht nur unterschiedliche Krankheitsverständnisse und -konzepte, auch unterschiedliche Vorstellungen in der Behandlung und vor allem im Umgang mit dem Kranken können zu Missverständnissen, Unverständnissen, Befremden und Ablehnungen führen.

Zur Verdeutlichung soll auf Ausführungen von Peseschkian verwiesen werden (9). In seiner transkulturellen Betrachtung zwischen Orient und Okzident beschreibt er folgende Umgangsformen der Umgebung und Wahrnehmungen des Kranken. Orient: „Ist hier jemand erkrankt, so wird das Bett ins Wohnzimmer gestellt. Der Kranke steht im Mittelpunkt des Geschehens und wird von zahlreichen Familienmitgliedern, Verwandten und Freunden besucht. Ein Ausbleiben der Besucher würde als Beleidigung und mangelnde Anteilnahme aufgefasst.“ Okzident: „Wenn jemand krank ist, möchte er seine Ruhe haben. Er wird von wenigen Personen besucht. Besuche werden auch als soziale Kontrolle empfunden.“

Ein weiteres Beispiel stellt der Umgang mit dem Tod und der Trauer dar.

- Im Orient besuchen 8 bis 40 Tage lang alle Verwandte, Freunde, Bekannte und andere Mitmenschen die Hinterbliebenen und geben ihnen so das Gefühl der Geborgenheit. Geteiltes Leid ist halbes Leid.

- Im Okzident ziehen sich die Betroffenen dagegen häufig zurück.

Ein weiterer Unterschied liegt darin, dass mediterrane Patienten die Krankheit häufig als etwas betrachten, das in den Körper eindringt und Besitz von ihm ergreift. Übernatürlich orientierte Erklärungs- und Handlungsmuster sind bei ihnen nicht selten anzutreffen. Magische Vorstellungen bilden Erklärungsmodelle für Störungen im Verhältnis zu den Mitmenschen (10). So können z.B. Böser Blick, Verzauberung, Besessenheit, Verwünschung mit dem Gefühl der Bedrohung einhergehen. Zudem können sie die Unfähigkeit aufzeigen, sich gegen andere abzugrenzen.

Das Tragen von blauem Auge bzw. blauem Stein (tr.: nazarboncugu) oder Amuletten sind Versuche, den Bösen Blick, die Verzauberung oder die Verwünschung aufzulösen, abzuwenden oder vorzubeugen (10).

Während die moderne westliche Medizin eine Trennung von Psyche und Soma praktiziert, existiert hier ein ganzheitliches Krankheitskonzept. Die körperlich-seelische Unbehaglichkeit äußert sich in ganzheitlicher Befindlichkeitsstörung. Bei einem soziokulturellganzheitlichem Krankheitsgefühl fällt es schwer, klar abgrenzbare Symptome anzugeben. Es werden daher häufig allgemeine Umschreibungen wie Erschöpfungs- und Verstimmungszustände, Insuffizienzgefühle oder Schmerzzustände vorgetragen (15).

Durch diese Erläuterungen sollen die Komplexität und die kulturelle Vielfalt in der Gesellschaft, in der wir uns befinden, vor Augen geführt werden. Vor diesem Hinter- grund stellt sich nun die Frage, sind die psychiatrischen Regeldienste auf diese multikulturelle Realität eingestellt und vorbereitet?

\section{Wie könnte die interkultu- relle Versorgung umgesetzt} werden?

Im interkulturellen Beratungsund Behandlungsprozess sollte aus genannten Gründen in jedem Fall die Kommunikation möglich sein. Darüber hinaus sollten Kenntnisse über kulturelle, religiöse und soziale Hintergründe und auch der verbalen und nonverbalen Sprache mit ihren semantischen und sinnbildlichen Eigenschaften als Grundlagen verfügbar sein (15). Es ist bekannt, dass Sprache die Befindlichkeit zum Ausdruck bringt und Träger von Emotionen und Identität darstellt. Die Sprache ist verbunden mit Selbstwertgefühl und Selbstbewusstsein $(4,15)$.

Der Einsatz von professionellen Übersetzern als Vermittler von Sprache und Kultur kann somit die interkulturelle Kommunikation ermöglichen (15).

Vor dem Einsatz von sog. „Zufallsdolmetschern“ wie Rückgriff auf Kinder, Partner, Reinigungskräfte sei gewarnt, da er erhebliche Fehlerquellen verbirgt und zu Überforderungen führen kann.

Neben dem routinemäßig zu fordernden Einsatz von Sprach- und Kulturvermittlern, wenn „Sprachlosigkeit" vorliegt, sollten bei allen Mitarbeitern der psychiatrischen Versorgungssysteme interkulturelle Kompetenz bestehen.

Diese beinhaltet, dass zunächst einmal die Mitarbeiter bereit sind, die eigene Person und Position zu reflektieren, sich insbesondere klar darüber zu werden, dass sie selbst nicht losgelöst von kulturellem Kontext sind. Sie sollten über die Bereitschaft verfügen, sich Kenntnisse über soziokulturelle Hintergründe von Patientinnen und Patienten, mit denen sie in eine therapeutische Beziehung eintreten, anzueignen. Es kommt in diesem Zusammenhang darauf an, dieses Wissen bzw. diese Kenntnisse zu reflektieren, um nicht in stereotypen Annahmen zu verbleiben $(5,6)$. 
Dabei kann auch sehr hilfreich sein, die Betroffenen und/oder seine Familie als „Fachleute“ für die jeweilige Kultur direkt anzusprechen und u.a. zu fragen, wie die jeweils vorliegenden Symptome, Beschwerden, Reaktionen oder Phänomene im eigenen kulturellen Kontext verstanden bzw. gewertet werden, was es im jeweiligen Kontext bedeutet, dieses Problem zu haben, und ob der Betroffene und/oder sein Umfeld Konsequenzen zu befürchten haben. Dabei kann es sich schwierig gestalten, kulturelle Aspekte sowie Informationen angemessen, ohne Über- oder Unterbewertung oder gar Verleugnung, einzuordnen.

Eine weitere Möglichkeit zur Integration von Migranten stellt auch die Einstellung von muttersprachlichen Mitarbeitern dar. Die Schwelle der Inanspruchnahme von Einrichtungen sinkt, wenn ein Migrant dort beschäftigt ist, unabhängig von der Herkunft (12).

\section{Schlussfolgerung}

Obwohl die Migration in der heutigen Gesellschaft einen dauerhaften und zunehmenden Prozess darstellt und nie abgeschlossen sein wird, ist die Integration der Migranten in das bestehende psychiatrischpsychotherapeutische Versorgungssystem nach wie vor defizitär. Diese Integration muss politisch gewollt, finanziert und entsprechend auch durchgesetzt werden.

Dabei geht es um die Ergänzung von migrationsspezifischen Strukturen in die bereits bestehende Einrichtungen der Versorgungsdienste, und nicht um ihre Neuschaffung. Insellösungen, wie sie bislang anzutreffen sind, können nur eine Übergangslösung darstellen (12).

\footnotetext{
Although people with migration background belong to the psychiatric health care system in the last decades, their consultation, care and treatment is still deficient. By intercultural opening of medical care facilities an important factor for integration could be possible. Intercultural competence is an essential condition thereby. Aim should be to face all patients with the same consistent standards.
}

Keywords: People with migration background - psychiatric health care systems - intercultural opening - intercultural competence - consistent standards

\section{Literatur}

1. Beauftragte der Bundesregierung für Ausländerfragen. Daten und Fakten zur Ausländersituation 20. Auflage, Februar 2002.

2. Brucks U, Wahl WB,. Über-, Unter-, Fehlversorgung? Bedarfslücken und Strukturprobleme in der ambulanten Gesundheitsversorgung für Migrantinnen und Migranten. In: Borde T, David Matthias (Hrsg.). Gut versorgt? Migrantinnen und Migranten im Gesundheits- und Sozialwesen. Frankfurt am Main, Mabuse-Verlag GmbH, 2003, 15-34

3. Ete E. Ethnomedizinische Aspekte der Interaktion mit türkischen Patienten. In: Koch E, Özek M, Pfeiffer WM (Hrsg.): Psychologie und Pathologie der Migration Deutsch-türkische Perspektiven, Band 1 der Schriftenreihe der Deutsch-Türkischen Gesellschaft für Psychiatrie, Psychotherapie und Psychosoziale Gesundheit e. V., 1995, 209-216

4. Haasen C. Kultur und Psychopathologie. In: Haasen, C., Yagdiran, O. (Hrsg.). Beurteilung psychischer Störungen - in einer multikulturellen Gesellschaft, LambertusVerlag, Freiburg im Breisgau, 2000: 13-28

5. Hegemann T. Interkulturelles lernen. Ein multidimensionaler Ansatz zum Erwerb interkultureller Kompetenzen. In: Hegemann T, Salman R (Hrsg.): Transkulturelle Psychiatrie. Konzepte für die Arbeit mit Menschen aus anderen Kulturen, Psychiatrie Verlag, Bonn, 2001: 191-205

6. Hegemann T. Transkulturelle Kommunikation und Beratung. Die Kompetenz, über kulturelle Grenzen hinweg zu kommunizieren. In: Hegemann T, Salman R (Hrsg.) Transkulturelle Psychiatrie. Konzepte für die Arbeit mit Menschen aus anderen Kulturen, Psychiatrie Verlag, Bonn, 2001, 116-129

7. Koch E. Biographien türkischer Rentenbewerber. In: Collatz J et al. (Hrsg.): Transkulturelle Begutachtung. VWB - Verlag für Wissenschaft und Bildung, Berlin, 1997 37-48

8. Koptagel-llal G. Migration und Trauma. Zur Psychodynamik der Konflikte und Traumen in der Migrationssituation. Vortrag auf dem 2. Internationalen Kongress zum Thema Körper - Seele - Trauma vom 11.3. bis 14.3.1999 in Göttingen

9. Peseschkian N. Die Notwendigkeit eines transkulturellen Austausches. Dargestellt am transkulturellen Aspekt der Positiven Psychotherapie. In: Heise T (Hrsg.) Transkulturelle Psychotherapie - Hilfen im ärztlichen und therapeutischen Umgang mit ausländischen Mitbürgern. Transkulturelles Psychoforum 4. VWB, Verlag Wissenschaft und Bildung 1998, 195-210

10. Pfeiffer MW. Organbezogene Angstzustände und Befürchtungen. In: Pfeiffer MW (Hrsg. Transkulturelle Psychiatrie, 2. Neubearbeitete und erweiterte Auflage, Sammlung psychiatrischer und Neurologischer Einzeldarstellungen. Stuttgart, Georg
Thieme Verlag Stuttgart 1994: 90-97

11. Schepker R. Anderssein - das Eigene und das Fremde. In: Bundesarbeitsgemeinschaft Leitender Mitarbeiter-/innen der Pflege und Erziehungsdienste kinder- und jugendpsychiatrischer Kliniken und Abteilungen (Hrsg.). Anderssein - Anders behandeln. Arbeitstherapie Druck \& Papier des Nds. Landeskrankenhauses Hildesheim, 2000: $35-64$

12. Schouler-Ocak M. Kultursensibles Angebot für türkeistämmige Patienten in der Regelversorgungseinrichtung Institutsambulanz des NLKH Hildesheim. In: Koch E, Schepker R, Taneli S (Hrsg.). Psychosoziale Versorgung in der Migrationsgesellschaft. Deutsch -Türkische Perspektiven. Band 3 der Schriftenreihe der Deutsch-Türkischen Gesellschaft für Psychiatrie, Psychotherapie und psychosoziale Gesundheit e.V. (DTGPP). Lambertus-Verlag. Freiburg im Breisgau 2000, 68-79

13. Schouler-Ocak M. Migration und Trauma: Soziokulturelle Besonderheiten bei der ethnischen Minorität türkischer Herkunft. Familiäre, religiöse und kulturelle Gegebenheiten türkischer Familien. In: Bundesarbeitsgemeinschaft Leitender Mitarbeiter-/innen der Pflege und Erziehungsdienste kinder- und jugendpsychiatrischer Kliniken und Abteilungen (Hrsg.). Anderssein - Anders behandeln. Arbeitstherapie Druck \& Papier des Nds. Landeskrankenhauses Hildesheim 2000: 65-93

14. Sluzki EC. Psychologische Phasen der Migration und ihre Auswirkungen. In: Hegemann T, Salman R (Hrsg.): Transkulturelle Psychiatrie. Konzepte für die Arbeit mit Menschen aus anderen Kulturen. Bonn, Psychiatrie Verlag, 2001: 101-115

15. Tuna S, Salman R. Phänomene interkultureller Kommunikation im Begutachtungsprozess. In: Collatz J, Hackhausen W, Salman $\mathrm{R}$ (Hrsg.): Begutachtung im interkulturellen Feld. Zur Lage der Migranten und zur Qualität ihrer sozialgerichtlichen und sozialmedizinischen Begutachtung in Deutschland. VWB - Verlag für Wissenschaft und Bildung, Reihe Forum Migration Gesundheit Integration Band 1, Berlin, 1999: 179-188

\section{Korrespondenzadresse}

Dr. med. Meryam Schouler-Ocak

Niedersächsisches Landeskrankenhaus Hildesheim

Akademisches Lehrkrankenhaus der GeorgAugust-Universität Göttingen

Goslarsche Landstraße 60

31135 Hildesheim

mocak@web.de 